\title{
Design considerations for a digital feedback system to control self-bunching in ion-storage rings
}

\author{
V. Ziemann \\ The Svedberg Laboratory, 75121 Uppsala, Sweden \\ (Received 27 February 2001; published 30 April 2001)
}

\begin{abstract}
We discuss the feasibility of a digital feedback system to cure self-bunching of the electron-cooled coasting ion beam in ion-storage rings such as CELSIUS [S. Holm, A. Johansson, S. Kullander, and D. Reistad, Phys. Scr. 34, 513-532 (1986)]. Such a system is based on a fast digital filter that acts as a tunable artificial wake potential. It may also aid stable operation of accumulator rings for future spallation neutron sources or heavy ion rings used for inertial fusion energy production.
\end{abstract}

DOI: 10.1103/PhysRevSTAB.4.042801

PACS numbers: $29.27 . \mathrm{Bd}$

\section{INTRODUCTION}

The electron-cooled ion beam in ion-storage rings such as CELSIUS [1] frequently shows self-bunching which is typically interpreted as the beam operating outside the stability region [2] due to its high intensity and small energy spread. The sources of this instability are wakefields or, equivalently, impedances. The mechanism of the instability is that the beam excites electromagnetic fields in the beam pipe that affect subsequent sections of the beam. These latter sections of the beam in turn excite new fields and so on. This mechanism obviously forms a closed loop that can become unstable, which is undesirable in nuclear physics ion-storage rings, because it creates background for the experiments and it will contribute to particle losses in high intensity accelerators, thus increasing the radiation load.

The energy change $\Delta E(z)$ or wakefield a particle at longitudinal position $z$ experiences is determined by the longitudinal charge density $\rho\left(z^{\prime}\right)$ of the beam weighted by the wake potential $W\left(z-z^{\prime}\right)$ that depends on the distance $z-z^{\prime}$ between the affected particle and the section of the beam that excites the field

$$
\Delta E(z) \propto \int_{-\infty}^{z} \rho\left(z^{\prime}\right) W\left(z-z^{\prime}\right) d z^{\prime} .
$$

There are two major contributors to the wake potential in low-energy ion-storage rings [3]. First, the space charge impedance and, second, contributions from resonatorlike structures such as cavities and bellows. Even though the space charge impedance is purely capacitive and does not drive instabilities below transition, we will include it in the simulation. The real "culprits" that make the beam unstable and cause self-bunching are the resonatorlike wakes, and we will focus on canceling their adverse affect.

TABLE I. CELSIUS parameters.

\begin{tabular}{lc}
\hline \hline Circumference & $81.76 \mathrm{~m}$ \\
Rigidity & $1-7 \mathrm{Tm}$ \\
Revolution frequency (proton) & $1.1-3.3 \mathrm{MHz}$ \\
Revolution frequency (argon) & $0.5-2.6 \mathrm{MHz}$ \\
\hline \hline
\end{tabular}

To accomplish this, note that the expression for the energy loss given in Eq. (1) is almost identical to that of a finiteimpulse response (FIR) filter [4], where the input signal corresponds to the earlier charge density and the wake function $W(\Delta z)$ corresponds to the filter coefficients. It is intuitively appealing to try to compensate the adverse effect of the wake fields by a feedback system that records the beam intensity using the sum signal from a beam position monitor (BPM), then digitizes this signal at a high rate, passes the signal through a FIR filter, and feeds it back to the beam via an amplifier and a longitudinal kicker structure. We will show the feasibility and features of such a system in the remainder of this report.

Note that such an active feedback system bears some resemblance to the "SPEAR capacitor" discussed in Ref. [5], where a device with a specially tailored impedance was introduced in SPEAR in order to compensate adverse effects of wake fields. This device had limited success, possibly because the artificial wake was fixed. On the other hand, in a digital system, many different configurations can be easily tested by downloading new filter coefficients.

In the following sections we will discuss the simulation code results from simulations which will define the parameters of the feedback system that could be used in CELSIUS (see Table I), discuss technical issues, and offer a concluding summary.

\section{SIMULATION}

In order to make the simulation as realistic as possible we utilize a multiparticle tracking code that typically propagates 10000 particles in the longitudinal phase space. As phase space coordinates we choose the phase $\phi$ which is proportional to the arrival time at a given position in the ring and the relative energy offset with respect to a reference particle $\Delta p / p$. The unperturbed dynamics of the particles is given by

$$
\begin{aligned}
\phi_{n+1} & =\phi_{n}+\eta\left(\frac{\Delta p}{p}\right)_{n}, \\
\left(\frac{\Delta p}{p}\right)_{n+1} & =\left(\frac{\Delta p}{p}\right)_{n},
\end{aligned}
$$


where the subscript $n$ denotes the turn number and $\eta=$ $\alpha-1 / \gamma^{2}$ the phase slip factor. $\alpha=1 / \gamma_{T}^{2}$ is the momentum compaction factor, $\gamma_{T}$ is the transition energy, and $\gamma$ is the relativistic energy factor of the particle. Note that we use the convention that $\eta$ is negative below transition. In this way, particles with positive momentum $\Delta p / p$ are moving toward the left, when operating below transition. We also see from Eq. (2) that the energy of the unperturbed particle stays constant and particles with positive energy offset $\Delta p / p$ will move towards smaller phase values, provided that $\eta$ is negative.

Figure 1 shows output of the program LTRAK which runs the simulation and displays the particle distribution as the simulation progresses. In the main window in the top left corner, the longitudinal phase space is shown with phase between 0 and $2 \pi$ in the horizontal direction and relative momentum $\Delta p / p$ in the range $\pm 10 \times 10^{-3}$ in the vertical direction. The window below the main window shows the longitudinal distribution, i.e., the signal that the BPM sum signal would show. The window on the right shows the momentum distribution. The small window in the lower right corner displays the fraction of surviving particles and the bunching factor which is the absolute value of the first Fourier harmonic. Both quantities are plotted on a scale from zero to unity.

In the code, the wake potential is represented as a table with 256 entries that corresponds to the wake potential discretized over 256 points of a revolution. This requires that the wake has decayed over a revolution period or that the wake potential represents a "wrapped-around" version of

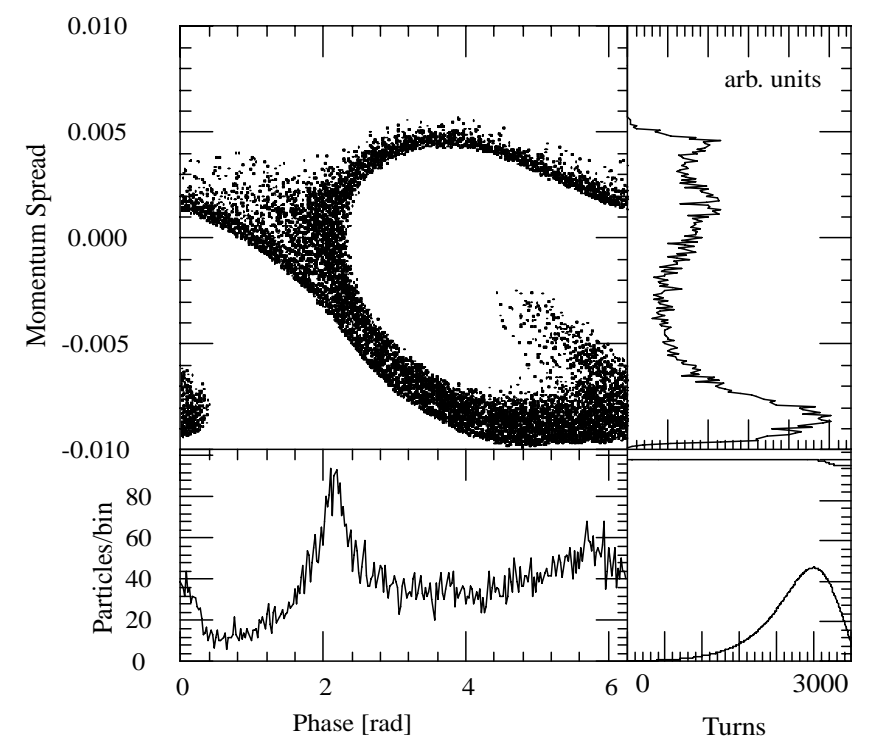

FIG. 1. The main window shows the longitudinal phase space after 3000 turns. The windows below and to the right show the longitudinal bunch distribution and the momentum distribution, respectively. The small window in the lower right-hand corner shows the survived fraction of particles (upper trace) and the bunching factor (lower trace) as a function of turn number. The vertical scale here extends from zero to unity. the wake potential. The latter case is only a valid approximation if the distribution function changes slowly with respect to the revolution period because, when calculating the effect of a slice of beam that lies a few turns, say, three turns, back, we effectively use the distribution of the previous turn. For the most part, the wakes are resonatorlike with impedances given by

$$
Z(\omega)=\frac{R_{s}}{1+i Q\left(\omega / \omega_{r}-\omega_{r} / \omega\right)},
$$

where $R_{S}$ is the shunt impedance of the resonator, $Q$ is its quality factor, and $\omega_{r}$ is the resonance frequency. Fourier transforming this expression leads to the following wake potential:

$$
W(\tau)=e^{-C \tau}[A \cos (D \tau)-B \sin (D \tau)],
$$

with $A+i B=\omega_{r} R_{S}\left(1+i / \sqrt{4 Q^{2}-1}\right) / Q$ and $C-$ $i D=\omega_{r}\left(1-i \sqrt{4 Q^{2}-1}\right) / 2 Q$. Note that the wake potential at the origin has the magnitude $\omega_{r} R_{s} / Q$, which translates into an energy loss of a deltalike charge distribution with charge $N e$ by $\Delta U=N e \omega_{r} R_{s} / Q$.

The simulation of the space charge wake deserves some special attention. From Ref. [2] we know that the longitudinal electric field due to space charge fields in a perfectly conducting beam pipe is given by

$$
E_{s}=-\frac{e(1+2 \ln b / a)}{4 \pi \varepsilon_{0} \gamma^{2}} \frac{\partial \lambda}{\partial s},
$$

where $\lambda$ is the longitudinal charge density in particles $/ \mathrm{m}$, $a$ is the transverse beam size, and $b$ is the beam pipe radius. $\varepsilon_{0}=1 / Z_{0} c$ is the dielectric constant and $Z_{0}$ is the impedance of free space $(377 \Omega)$. In the simulation, we calculate the longitudinal charge distribution by binning the particles after every turn. The energy loss $U_{i}$ for particles in bin number $i$ due to space charge is then given by

$$
\Delta U_{i}=Z_{\mathrm{sc}} \frac{I_{i+1}-I_{i}}{2 \pi / M},
$$

where $Z_{\mathrm{sc}}=Z_{0}(1+2 \ln b / a) / 2 \beta \gamma^{2}$ is the space charge impedance, $M=256$ is the number of longitudinal bins in one turn, and $I_{i}$ is the current in bin number $i$. For a homogenous coasting beam, we have $I_{i}=I_{\text {total }} / M$ for all $i$. If we consider $48 \mathrm{MeV}$ protons in CELSIUS, the space charge impedance is about $10 \mathrm{k} \Omega$, and if we assume that each of the macroparticles represents $1 \mu \mathrm{A}$ we have $\Delta U \approx 0.4 \mathrm{eV} \times\left(N_{i+1}-N_{i}\right)$. It is also instructive to calculate the Fourier transform of the space charge wake where one obtains a function linear in frequency, which means that the space charge wake acts like a high pass filter. As a matter of fact, this behavior is not so surprising because the term $N_{i+1}-N_{i}$ looks like a discrete version of the derivative of a Dirac-delta function of which the Fourier transform is known to be a linear function in frequency. Including space charge will thus make the wake more noisy. 
In the simulation we have adjusted the space charge wake function and the resonator wake such that the contribution of the wake at the origin from space charge is 10 times that of the resonator wake. Varying this ratio showed little influence on the growth rate. The resonator wake was chosen to be a $Q=1$ resonator at the fundamental revolution harmonic with a strength such that the beam becomes unstable within 3000 turns in order to economize on the simulation time.

It is interesting to observe the behavior of the bunching factor as the instability develops in the small windows at the bottom right of the main panel. As can be seen in Fig. 1, it shows exponential growth before saturating in the last quarter of the simulation. In Fig. 2 we plot the logarithm of the bunching factor as a function of the turn number together with a linear least squares fit in a selected region. The growth rate, which is 451 turns in this case, is displayed at the top of the graph. The growth rate will be used exhaustively as a figure of merit when analyzing the performance of a feedback system in the remainder of this paper.

In the model we introduce a feedback system by using the sum signal of the BPM, which corresponds to the longitudinal projection of the distribution, and passing it through a FIR digital filter whose filter coefficients can be specified by writing values to an array. The resulting kicks are multiplied by a gain factor and written to a ring buffer that keeps track of the feedback kicks for the last eight turns with 256 kicks per turn. By picking kicks from the ring buffer after a variable time delay and applying them to the particles, it is possible to simulate the effect of a FIR-based feedback system on the beam. In a real implementation a buffer depth corresponding to two turns should suffice in order to allow adjusting the delay time over the range of a full revolution period.

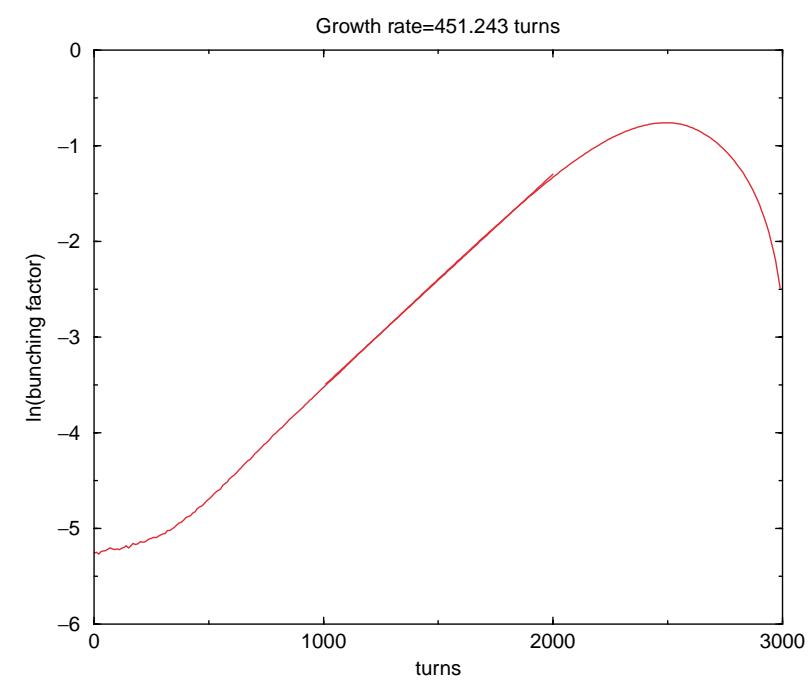

FIG. 2. (Color) The logarithm of the bunching factor as a function of the turn number. A linear least squares fit to the slope in a finite region yields the growth rate of the mode that is unstable.
In the same way as for the wake potential, it is possible to specify the filter coefficients, where one can choose low, high, bandpass, or bandstop filters, load coefficients from a file, or copy the wake potential. Other features include digitizing the filter in time, which means applying a zero-order hold on a data value for a number of samples. Moreover, digitizing in amplitude is also implemented in order to simulate finite word lengths if the filter is implemented on a fixed point digital signal processor (DSP) or programmable logic.

\section{RESULTS}

Initial tests with various filter types, such as band or lowpass, were rather unsuccessful in reducing the growth rate of the instability. Finally, we realized that we could compensate the effect of the wake as given in Eq. (1) by using the wake function as filter coefficients for the FIR filter and adjust the gain of the feedback in such a way that the kick applied to the beam is equal to that from the wake, thus creating an artificial wake that compensates the real one. Figure 3 shows the growth rate of the instability as a function of the feedback gain when the filter coefficients are chosen to be the same as the coefficients that define the wake function. In Fig. 4 we show the kicks that the beam gets from the wake (solid line) and from the feedback (dashed line) as a function of position inside the ring when the feedback gain is adjusted to half that of the wake. Observe that the magnitude of the kicks from the feedback is about half that of the wake, and the shape of the wake kicks is closely followed by that of the feedback kicks, except for the opposite sign. Furthermore, the wake is more noisy due to the influence of the space charge component, which is not included in the feedback coefficients.

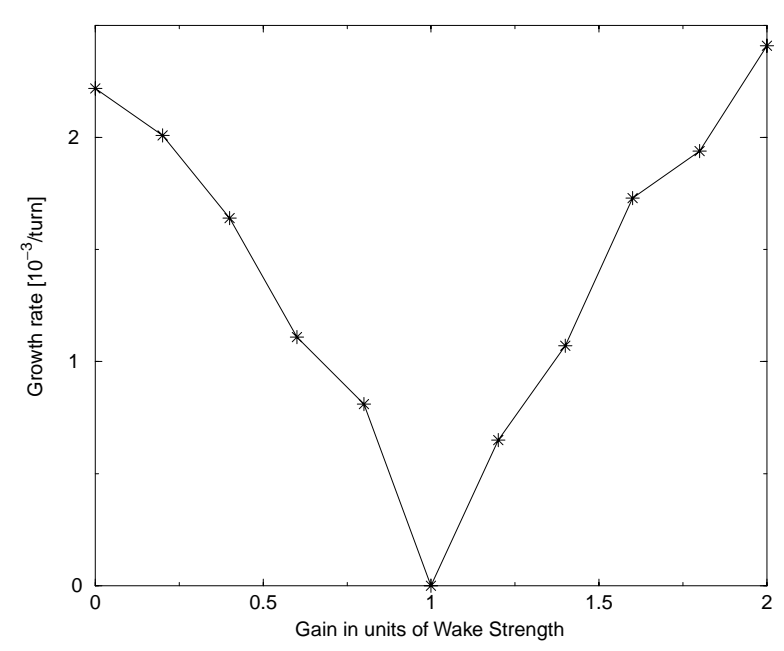

FIG. 3. Growth rate of the bunching factor as a function of the gain of the feedback system. The filter coefficients are identical to the wake function $(Q=1$ resonator at first harmonic plus space charge). Note the perfect cancellation when the gain is identical to the strength of the wake. 


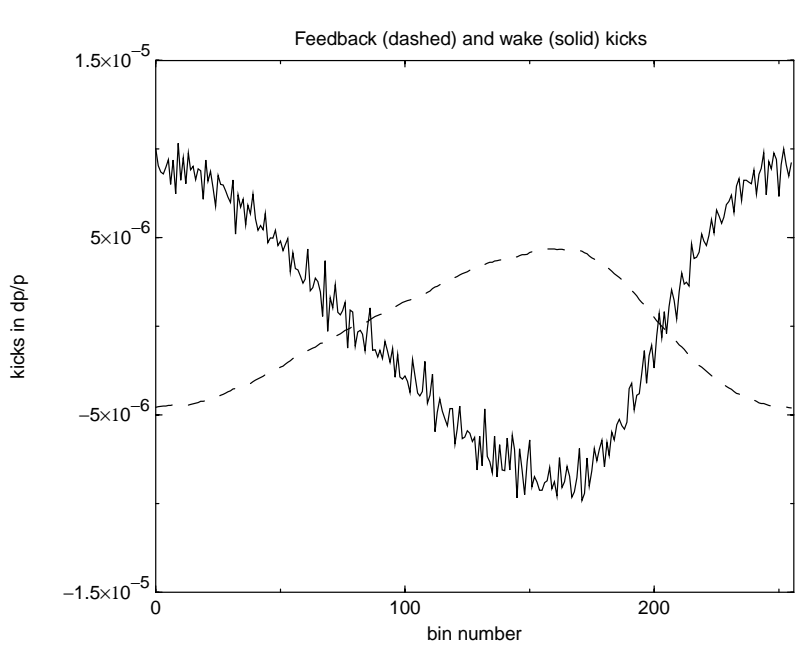

FIG. 4. The kicks from the wake and the feedback. Note that the feedback kicks are just the negative of the wake kicks.

In the previous section we applied the feedback kicks a multiple of the revolution time after we applied the wake kicks. In practice we do not know when and where in the ring the wake kicks hit the beam and, in a practical implementation, we need to control the delay between picking up the BPM data and kicking the beam. This delay parameter will then have to be optimized experimentally. In order to investigate the sensitivity of the feedback on this parameter we run the code and vary the delay time at which the feedback kicks are taken from the ring buffer. In Fig. 5 we show the growth rate as a function of the delay time, represented here as a phase offset where $360^{\circ}$ corresponds to the full revolution period and negative phase means longer delay. Zero degrees means that the feedback kicks are applied an integer number of revolution periods after the wake kicks. For unexplained reasons, the growth rate has an asymmetric behavior. However, we can observe that a delay that is slightly too large (negative phase) will cause the growth rate to increase dramatically, whereas a decrease of the delay by $35^{\circ}$ will still prevent the startup of the instability. In any case, we have a window of about $40^{\circ}$ in which the feedback is functioning well, which means that the tolerance for this parameter is rather loose. For CELSIUS at injection energy the revolution time is about $1 \mu \mathrm{s}$, and the tolerance for the delay is thus on the order of 100 ns. Furthermore, we found that shifting the delay time by multiples of the revolution time does not significantly change the behavior of the feedback system. This can be attributed to the fact that the longitudinal beam distribution changes rather slowly on the time scale of several 100 turns, whereas the delay time is varied over only a few turns.

So far, we always employed filter coefficients that are given as floating point numbers. In a real system, where the filter calculations have to be made at a rate of a few tens of $\mathrm{MHz}$, we can gain some speed by reducing the word length in the calculation. We simulate this by digitizing the filter

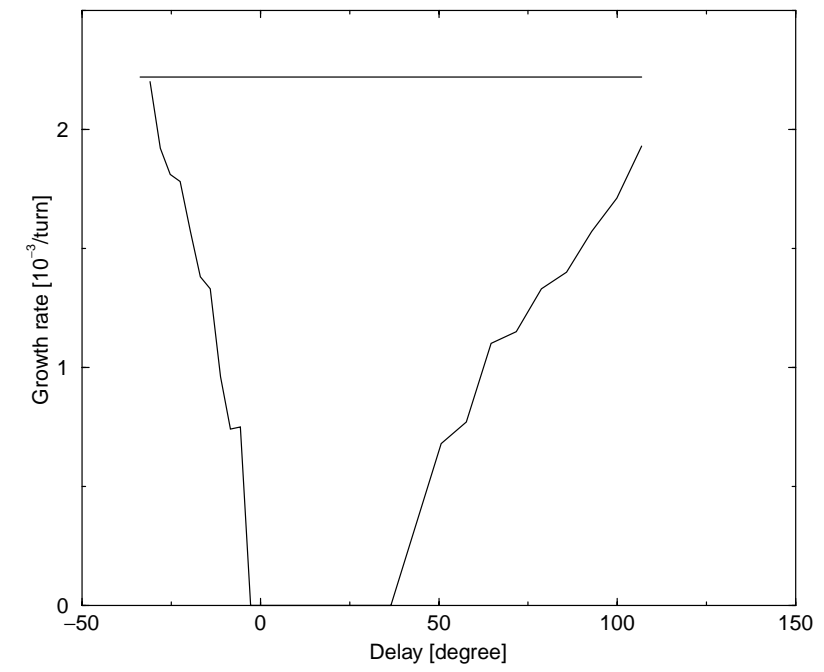

FIG. 5. The growth rate of the bunching factor as a function of the time delay given as phase $\left(360^{\circ}\right.$ corresponds to the revolution time). The horizontal line denotes the growth rate in the absence of feedback.

coefficients in steps of powers of 2 and found that only the average energy loss is changed, which can be easily compensated by adding a constant to the filter coefficients. The growth rate, on the other hand, is not affected even if the filter coefficients are digitized in eight steps, that is 3 bit. Using a low number of bits makes it possible to use a lookup table approach for the multiplications needed in the FIR filter calculations, provided the number of bits used in the digitization of the BPM signal is also small. However, in order to be on the safe side, we will try to use 6 or 8 bits in the analog-to-digital converter (ADC) and filter coefficients which will also make matching the dynamic range of the BPM and the ADC less critical.

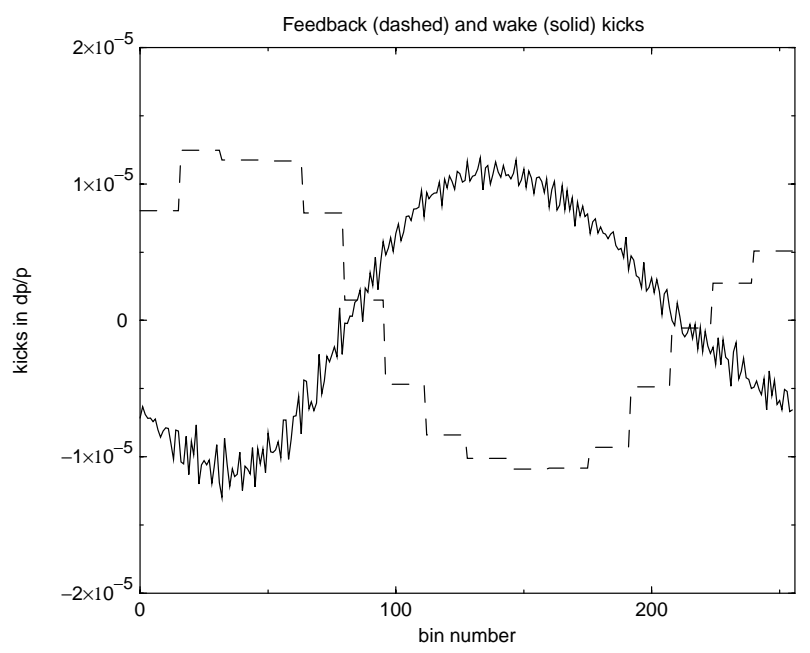

FIG. 6. The kicks from the wakefield and the feedback system for the case where the feedback kicks the beam 16 times per turn. 
Next we discuss the effect of sampling and processing the signals at a lower speed. In the preceding sections we always used 256 sample points per turn for the beam intensity, the wake, and the filter coefficients. Since this would correspond to using a $256 \mathrm{MHz}$ data processing system when running at a revolution frequency of $1 \mathrm{MHz}$ it would pose a high demand on the ADCs and filters. In order to ease these requirements we investigate the effect of operating at lower processing speeds. In the code we have implemented sampling and processing at a lower rate by using only every $n$th sample of the beam intensity signal and also updating the feedback-kick ring buffer only every
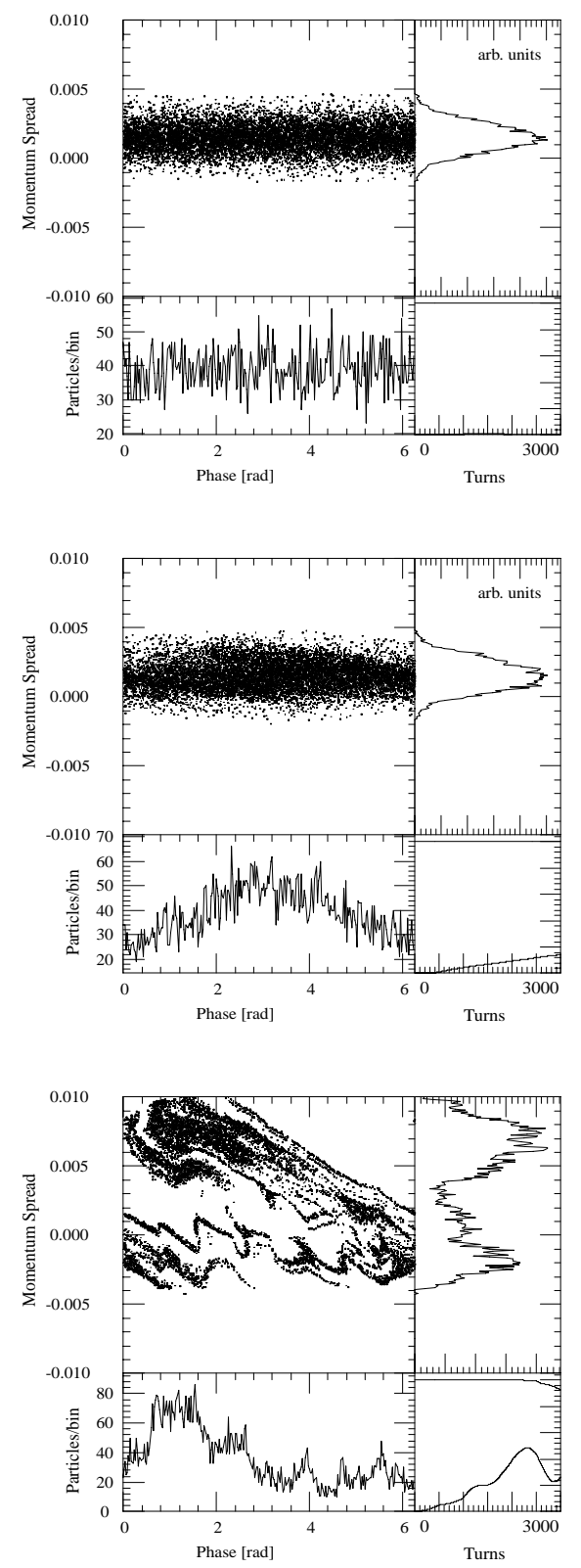

$n$th sample, which is then held constant for $n$ samples. An example of the kicks applied to the beam after 3000 turns for the case where only 16 kicks per turn were applied is shown in Fig. 6. Observe that the feedback kicks come in steps which are 16 bins long, whereas the kicks from the wake field are quasicontinuous.

Judging the performance degradation due to a slower feedback system using the bunching factor as a figure of merit proved difficult. In Fig. 7 we therefore choose to show phase space after 3000 turns for feedback systems operating at different sampling speeds. In the top left corner we show phase space when the beam was kicked
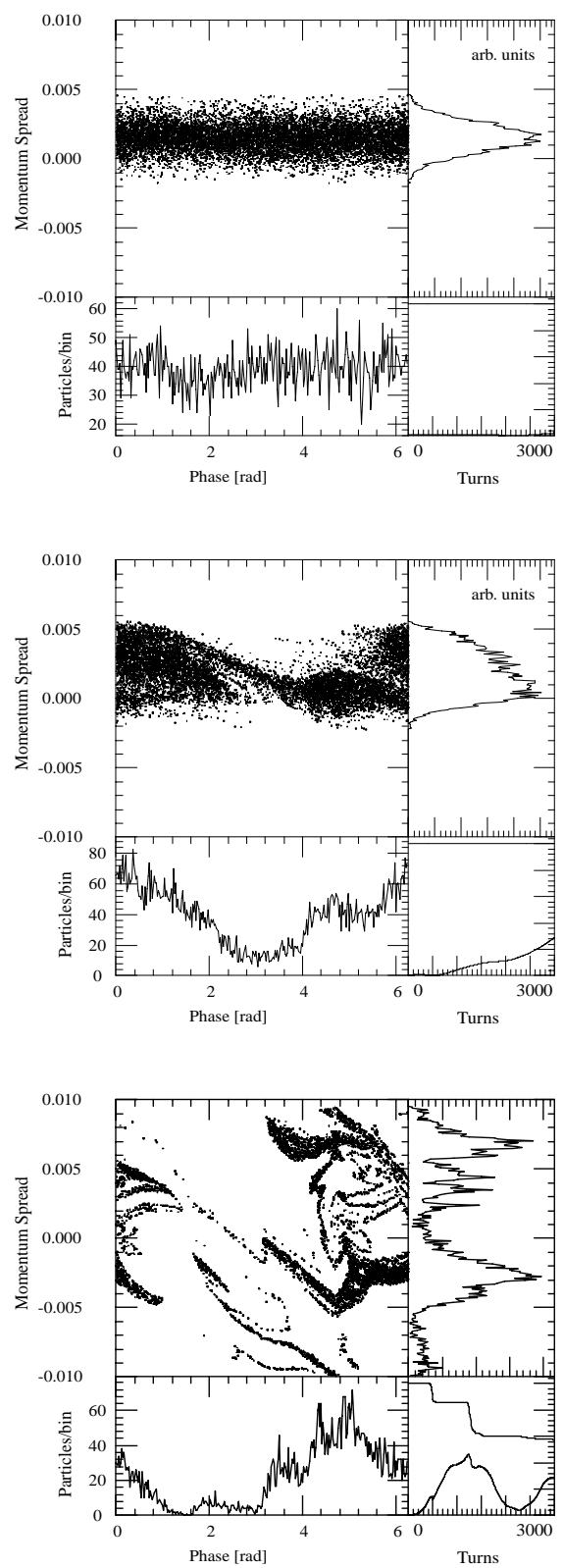

FIG. 7. The individual pictures show the longitudinal phase space after 3000 turns with 10000 particles with wake and feedback. The feedback uses $128 \mathrm{kicks} /$ turn (top left), $64 \mathrm{kicks} /$ turn (to the right), down to 4 kicks/turn (bottom right) in steps of the power of 2. Compare to the corresponding system without feedback in Fig. 1. 
128 times per turn (the kick is held constant for two consecutive beam slices as described above) and the number of kicks per turn was reduced in steps of factors of 2 down to 4 kicks/turn. In the top row we have 128 and 64 kicks per turn, in the second row 32 and 16 kicks per turn, and in the bottom row 8 and 4 kicks per turn. We observe from the longitudinal profile - the projection in the lower part of the respective pictures - that the bunching gets progressively worse as we reduce the number of kicks per turn, but also that the momentum spread - the width of the vertical projection on the right of the respective pictures increases. It appears that the first three distributions are tolerable, but the last three are not, because the energy spread increases dramatically. In any case, we see when
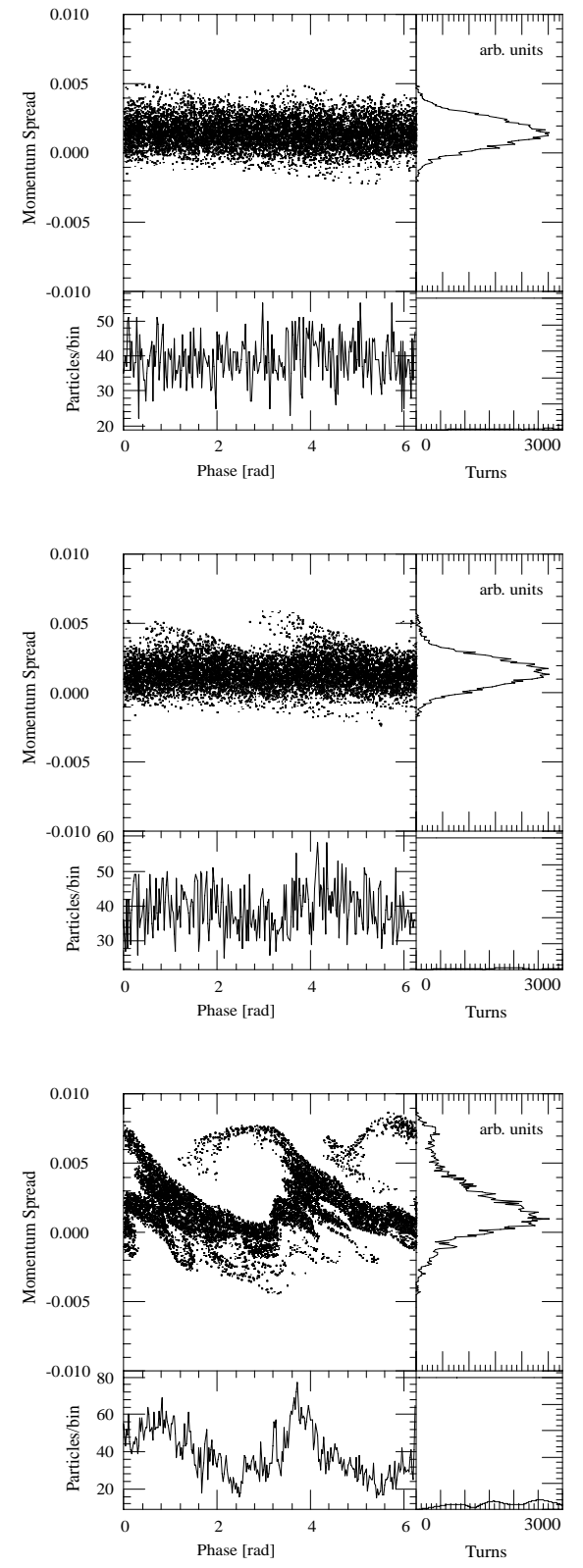

comparing to Fig. 1 that the feedback is beneficial in all cases with the exception of those in the bottom row.

When experimenting with different parameters, we found that we could improve the performance of the feedback system by decreasing the delay time or, equivalently, increasing the phase (see Fig. 5) by about $35^{\circ}$. The pictures with reduced delay time corresponding to those displayed in Fig. 7 are shown in Fig. 8. We observe that self-bunching is somewhat reduced and that the momentum spread in the right-hand picture in the second row is considerably smaller compared to that in Fig. 7. This means that we can operate a feedback system which only samples the beam at a rate which is 16 to 32 times the revolution frequency. In CELSIUS, at injection energy,
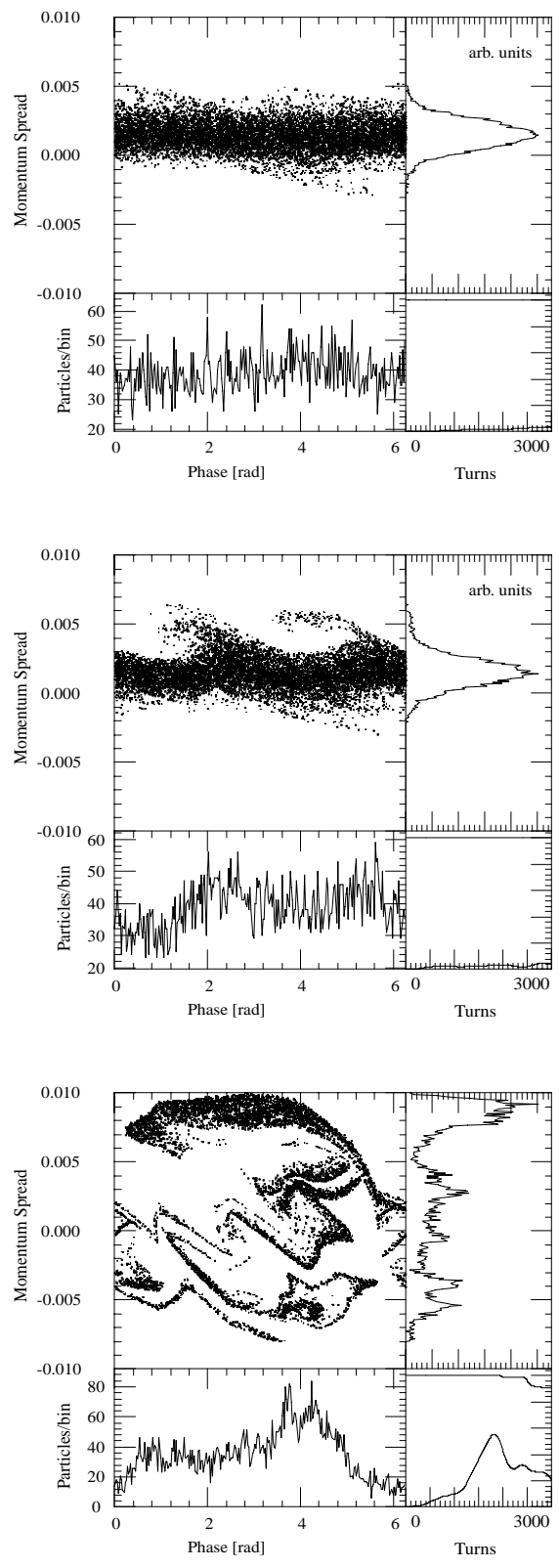

FIG. 8. The pictures corresponding to those in Fig. 7 where the feedback delay was reduced by a tenth of a revolution $\left(\approx 35^{\circ}\right)$. 
this means we can operate the feedback at about $30 \mathrm{MHz}$. This drastically relieves the demand on the ADC and the FIR filter.

During the investigation described in the previous paragraph, we observed that the average energy applied to the beam by the feedback is considerable and the beam's distribution moves up or down in phase space. This behavior can, however, be compensated by subtracting or adding a constant to the filter coefficients.

The required power can be estimated from the amplitude of the kick applied to the beam in order to compensate the growth of the instability for a given bunching factor. In the simulation, the growth rate of the uncompensated instability is about 500 turns, or about $0.5 \mathrm{~ms}$. The peak-to-peak value of the wake kicks is about $\Delta p / p \approx 10^{-6}$ when the bunching factor is $1 \%$. This translates to an energy change of about $\pm 1 \mathrm{kV}$ delivered to the beam. If the growth rate of the instability is smaller, the required voltage is reduced proportionally. This also translates into a reduced current that can be stored without self-bunching.

Next, we investigate the effect of feedback amplifier power saturation on the behavior of the feedback system. To this end we use the standard simulation scenario and limit the maximum kick that the feedback can deliver to the beam. We find that the feedback can prevent the instability from starting as long as fluctuations are small. Once a fluctuation has created a sufficiently large inhomogeneity, the feedback is, however, unable to contain it. The details are a function of the instability's growth rate and the amount of fluctuation in the beam.

A practical difficulty comes from the inability to accurately know the shape of the wake function. In a real implementation we have to guess the shape, which invariably leads to differences between the real wake that drives the instability and the filter coefficients that constitute the artificial wake. We are thus interested in the deterioration of the performance of the feedback system when there is a mismatch between the frequency or $Q$ value of the wake and the feedback. Since the wake functions for different frequencies (harmonics) look rather similar, we tested to correct a wake on the first harmonic with a feedback filter operating on the second harmonic, but that did not work at all. So, as a rule, the harmonic of the wake and the feedback have to agree. In the next step we try to understand the performance of the feedback system if there is a mismatch of the $Q$ value between the wake and the feedback system. To this end, we set up the wake to be a $Q=5$ wake at the first harmonic, add the space charge wake, and then try to compensate with a feedback system that operates at the first harmonic but vary the $Q$ value between 1 and 10. In almost all cases it is possible to compensate the instability almost perfectly. We see considerable bunching on the second harmonic only when we have a feedback system with $Q=1$. The first harmonic is properly damped here as well. This is not so surprising because the $Q=1$ resonator is rather broadband and even when it cancels the instability on the first harmonic it contains sufficient spectral power on the second to drive an instability there.

Having discussed the requirements and limitations of such a feedback system, we briefly present the relevant parameters that would be used in such a system for CELSIUS at injection energy with a revolution frequency around $1 \mathrm{MHz}$ : (i) $30 \mathrm{MHz}$ sample rate, (ii) 6- or 8-bit data word width, (iii) 60 filter taps (2 times sample rate over revolution frequency), and (iv) a few $100 \mathrm{~V}$ peak amplitude.

At peak energy in CELSIUS the revolution frequency is nearly tripled such that the sample rate is also tripled. Since self-bunching most often plagues low-energy beams, we will focus mainly on the low-energy scenario laid out above and will discuss technical implementation issues in the next section.

\section{PRACTICAL ISSUES}

The central item in the implementation of the feedback system is the FIR filter that serves as an artificial wake field and must operate at very high sample rates. There are recent developments in the field of programmable logic devices that make FIR filters operating above $100 \mathrm{MHz}$ with filter lengths of 256 taps, 16-bit word length possible [6]. As shown above, we need only 60 taps and word lengths of less than 8 bits at speeds of about $30 \mathrm{MHz}$ such that using a prototype board with a field programmable gate array, on-board ADC, and digital-to-analog converter (DAC) will be suitable [7]. These boards were originally designed for the telecommunication market, but can be equally well applied in accelerator-based research. In CELSIUS, the bandwidth of the front end of the BPM operates at bandwidths well over $50 \mathrm{MHz}$ so that we can directly use these signals. There is a longitudinal kicker structure in the ring that can be used to apply the kicks to the beam. The available voltage is about $\pm 60 \mathrm{~V}$ without problems and $\pm 120 \mathrm{~V}$ with some extra effort. The bandwidth of the kicker extends above $100 \mathrm{MHz}$ though its transfer function needs to be analyzed more carefully before doing an experiment. Delays in the system can be adjusted by the digital delay introduced above.

One of the first experiments with such a feedback system will be to use it as an artificial wake to make a low intensity beam unstable and observe the growth rate of the instability. It is important to check that the correct harmonic is excited and whether the growth rate scales with feedback gain in the manner expected from theory [2]. In this way the feedback system acts as an artificial wake function that can be used to investigate the accuracy of simulation codes for instabilities similar to that discussed in Ref. [3].

Once the expected behavior of the feedback system is established, one can use it to counteract self-bunching that occurs at higher beam currents. First, one has to observe at which harmonic self-bunching is most strongly visible by observing the zero-span signal of a BPM sum signal 
on a spectrum analyzer at different harmonics and determine which harmonic is worst. From the data of zero-span power versus time, we can determine the growth rate of the instability. From a snapshot of the longitudinal distribution, visible on a digital oscilloscope, we can deduce the bunching factor. Taken together this information can be used to estimate the strength $R / Q$ of the wake which, in turn, will determine the gain of the feedback system such that the magnitude of the applied kick is of the same order of magnitude. Since the magnitude of the $Q$ value is not critical, we would choose it to be about 5. Together with the knowledge of the dominant harmonic, this will allow us to specify the filter coefficients. The last parameter to set is the delay time of the feedback signal which can be adjusted to minimize the occurrence of self-bunching. Subsequent iterations of the parameters may be used to improve the situation further. Having stabilized one harmonic, one can repeat the procedure on the next stronger self-bunching harmonic.

\section{CONCLUSION}

We have determined the design parameters for a longitudinal feedback system in order to limit self-bunching instabilities in low-energy ion-storage rings such as CELSIUS. Such a feedback system is based on creating a digital artificial wake using an ADC, a fast FIR filter based on programmable logic, and a DAC. A BPM sum signal is fed into such a system, and the output voltage is applied to an amplifier feeding a longitudinal kicker structure. We found that sample rates of about $30 \mathrm{MHZ}$, data word lengths of 6 or 8 bits, and filter length of 60 taps are sufficient to stabilize the beam at injection energy in CELSIUS. We found that choosing the filter coefficients identical to those of the wake potential led to near perfect cancellation of self-bunching. A major drawback is that the wake potential is normally not known. In order to cure this deficiency we outlined a procedure that allows one to roughly determine the filter coefficients experimentally. If such a tuning procedure worked satisfactorily it could certainly be automated to make the feedback self-adapting, which would also constitute a measurement of the wake potential. But that remains to be seen after such a system has been built and tested.

[1] S. Holm, A. Johansson, S. Kullander, and D. Reistad, Phys. Scr. 34, 513-532 (1986).

[2] A. Hofmann, in Proceedings of the International School of Particle Accelerators, Erice, 1976, edited by M. H. Blewett (CERN, Geneva, 1977), p. 139-174.

[3] G. Rumolo, I. Hofmann, G. Miano, and U. Oeftiger, Nucl. Instrum. Methods Phys. Res., Sect. A 415, 411 (1998).

[4] E. Ifeachor and B. Jervis, Digital Signal Processing (Addison-Wesley, Reading, MA, 1993).

[5] K. L. Bane, M. H. R. Donald, A. Hofmann, J. Jowett, W. S. Lockman, P. L. Morton, R. Stege, W. L. Spence, and P. B. Wilson, in Proceedings of the First European Particle Accelerator Conference, Rome, 1988, edited by S. Tazzari (World Scientific, Singapore, 1989).

[6] C. Collins, Xcell 38, 67 (2000).

[7] DSP hardware accelerator product specification data sheets for GVA-200/220/250/270/290 available at www. gvassociates.com 